\title{
A dynamic electroviscoelastic problem with thermal effects
}

\author{
Sihem Smata and Nemira Lebri
}

\begin{abstract}
We consider a mathematical model which describes the dynamic process of contact between a piezoelectric body and an electrically conductive foundation. We model the material's behavior with a nonlinear electro-viscoelastic constitutive law with thermal effects. Contact is described with the Signorini condition, a version of Coulomb's law of dry friction. A variational formulation of the model is derived, and the existence of a unique weak solution is proved. The proofs are based on the classical result of nonlinear first order evolution inequalities, the equations with monotone operators, and the fixed point arguments.
\end{abstract}

Mathematics Subject Classification (2010): 74M15, 74M10, 74F05, 49J40.

Keywords: Piezoelectric, frictional contact, thermo-elasto-viscoplastic, fixed point, dynamic process, Coulomb's friction law, evolution inequality.

\section{Introduction}

Piezoelectricity is the ability of certain crystals, like the quartz, to produce a voltage when they are subjected to mechanical stress. On a nanoscopic scale, the piezoelectric phenomenon arises from a nonuniform charge distribution within a crystal unit cells, and the piezoelectricity is then perceived as the electrical polarization due to mechanical input. Different models have been developed to describe the interaction between the electric and mechanical fields (see, e.g.[12, 13, 15]). Therefore there is a need to extend the results on models for contact with deformable bodies which include coupling between mechanical and electrical properties. General models for elastic materials with piezoelectric effects can be found in $[13,17]$ and more recently in [2], viscoelastic piezoelectric materials in $[2,17]$ or elasto-viscoplastic piezoelectric materials have been studied in [9].

In this paper, we consider a general model for the dynamic process of frictional contact between a deformable body and a rigid obstacle. The material obeys an electro-viscoelastic constitutive law with piezoelectric and thermal effects. Moreover, the contact and friction are modelled by Signorini's conditions and a non local 
Coulomb's friction law. We derive a variational formulation of the model, which is set as a system coupling a variational second order evolution inequality. We establish the existence of a unique weak solution of the model. The idea is to reduce the second order evolution inequality of the system to first order evolution inequality. Then adopting fixed point methods frequently we prove an existence and uniqueness of displacement and temperature fields, using monotonicity and convexity properties. The importance of this paper is to make the coupling of an electro-viscoelastic problem with thermal effects. The paper is structured as follows. In Section 2 we describe the mechanical problem and provide comments on the contact boundary conditions. In Section 3 we list the assumptions on the data and derive the variational formulation. In Sections 4, we present our main existence and uniqueness results, which state the unique weak solvability of the Signorini's contact electro-visco -elastic problem with non local Coulomb's friction lawn conditions.

\section{Problem statement}

We consider a body made of a piezoelectric material which occupies the domain $\Omega \subset \mathbb{R}^{d}(d \leq 3)$ with a Lipschitz boundary $\Gamma$. The body is modelled with an electro-visco-elastic constitutive law, allowing piezoelectric effects. Let $[0 . T]$ be the time interval where $T>0$, let $\Gamma$ be split into three measurable parts $\Gamma_{1}, \Gamma_{2}$ and $\Gamma_{3}$ such that meas $\Gamma_{1}>0$. We assume that the body is fixed on $\Gamma_{1}$ and surface tractions of density $h$ act on $\Gamma_{2}$. On $\Gamma_{3}$, the body may come into contact with a rigid obstacle. In other hand, $\Gamma$ be split into two measurable sets $\Gamma_{a}$ and $\Gamma_{b}$ such that meas $\Gamma_{b}>0$ and $\Gamma_{3} \subset \Gamma_{b}$. We assume that the electrical potential $q_{0}$ act on $\Gamma_{a}$ and a surface electric charge of density $q_{2}$ act on $\Gamma_{b}$, we assume that the problem is quasistatic. The piezoelectric effect is the apportion of electric charges on surfaces of particular crystals after deformation. We denote by $\mathbb{S}^{d}$ the space of second order symmetric tensors on the space $\mathbb{R}^{d}$ and use $\cdot$ and $|$.$| for the inner product and the Euclidean norm on$ the space $\mathbb{R}^{d}$ (respectively; $\mathbb{S}^{d}$ ). Also $\nu$ represents the unit outward normal on $\Gamma$, the classical formulation of the electro-visco-elastic contact friction problem is described by:

Problem P. Find a displacement field $u: \Omega \times[0 . T] \rightarrow \mathbb{R}^{d}$, a stress field $\sigma: \Omega \times$ $[0 . T] \rightarrow \mathbb{S}^{d}$, an electric potential field $\varphi: \Omega \times[0 . T] \rightarrow \mathbb{R}$, an electric displacement field $D: \Omega \times[0 . T] \rightarrow \mathbb{R}^{d}$ and a temperature field $\theta: \Omega \times[0, T] \rightarrow \mathbb{R}_{+}$such that:

$$
\begin{gathered}
\sigma=\mathcal{A} \varepsilon(\dot{u})+\mathcal{G} \varepsilon(u)-\xi^{*} E(\varphi)-\theta M_{e} \text { in } \Omega \times[0 . T], \\
D=\beta E(\varphi)+\xi \varepsilon(u) \text { in } \Omega \times[0 . T], \\
\rho \ddot{u}=D i v \sigma+f_{0} \text { in } \Omega \times[0 . T] \\
\operatorname{div} D=q_{0} \text { in } \Omega \times[0 . T] \\
\dot{\theta}-\operatorname{div}(k \nabla \theta)=-M \nabla \dot{u}+q_{e} \text { in } \Omega \times[0 . T], \\
-k_{i j} \frac{\partial \theta}{\partial v} v_{j}=k_{e}\left(\theta-\theta_{R}\right) \text { on } \Gamma_{3} \times[0 . T] \\
\theta=0 \text { on } \Gamma_{1} \cup \Gamma_{2} \times(0, T), \\
u=0 \text { on } \Gamma_{1} \times[0 . T]
\end{gathered}
$$




$$
\begin{gathered}
\sigma_{\nu}=h \text { on } \Gamma_{2} \times[0 . T], \\
u_{\nu} \leq 0, \sigma_{\nu} \leq 0, u_{\nu} \sigma_{\nu}=0 \text { on } \Gamma_{3} \times[0 . T] \\
\left\{\begin{array}{l}
\left|\sigma_{\tau}\right| \leq \mu p\left|R \sigma_{\nu}\right| \\
\left|\sigma_{\tau}\right|<\mu p\left|R \sigma_{\nu}\right| \Longrightarrow \dot{u}_{\tau}=0 \\
\left|\sigma_{\tau}\right|=\mu p\left|R \sigma_{\nu}\right| \Longrightarrow \exists \lambda \geq 0 \text { such that } \sigma_{\tau}=-\lambda \dot{u}_{\tau} \quad \text { on } \Gamma_{3} \times[0 . T], \\
\varphi=0 \text { on } \Gamma_{a} \times[0 . T], \\
D \nu=q_{2} \text { on } \Gamma_{b} \times[0 . T], \\
u(0)=u_{0}, \dot{u}(0)=v_{0} \text { and } \theta(0)=\theta_{0} \text { in } \Omega \times[0 . T],
\end{array}\right.
\end{gathered}
$$

where (2.1), (2.2) are the thermo-electro -visco-elastic constitutive law of the material, we denote $\varepsilon(u)$ (respectively; $E(\varphi)=-\nabla \varphi, \mathcal{A}, \mathcal{G}, \xi, \xi^{*}, \beta$ ) the linearized strain tensor (respectively; electric field, the viscosity nonlinear tensor, the elasticity tensor, the third order piezoelectric tensor and its transpose, the electric permittivity tensor), $\theta$ represent the temperature, $M_{e}:=\left(m_{i j}\right)$ represents the thermal expansion tansor, (2.3) represents the equation of motion where $\rho$ represents the mass density, (2.4) represents the equilibrium equation, Equation (2.5) describes the evolution of the temperature field, where $k:=\left(k_{i j}\right)$ represents the thermal conductivity tensor, $q_{e}$ the density of volume heat sources. The associated temperature boundary condition is

given by (2.6), where $\theta_{r}$ is the temperature of the foundation, and $k_{e}$ is the exchange coefficient between the body and obstacle. Equation (2.7) means that the temperature vanishes on $\Gamma_{1} \cup \Gamma_{2} \times(0, T)$. We mention that Div $\sigma$, divD are the divergence operators, (2.8) and (2.9) are the displacement and traction boundary conditions, (2.10), (2.11) the Signorini's contact with a non local Coulomb's friction law conditions. $u$ ${ }_{\nu}$ and $u_{\tau}$ (respectively; $\sigma_{\nu}$ and $\sigma_{\tau}$ ) denote the normal displacement and the tangential displacement (respectively; the normal stress and the tangential stress). $R$ will represent a normal regularization operator that is a linear and continuous operator $R: H^{-\frac{1}{2}}(\Gamma) \rightarrow \mathbb{L}^{2}(\Gamma)$. We shall need it to regularize the normal trace of the stress which is too rough on $\Gamma . p$ is a non-negative function, the so-called friction bound, $\mu \geq 0$ is the coefficient of friction. The friction law was used in some studies with $p(r)=r_{+}$where $r_{+}=\max \{0, r\}$. Recently, from thermodynamic considerations, a new version of Coulomb's law is proposed, it consists to take:

$$
p(r)=r(1-\alpha r)_{+},
$$

where $\alpha$ is a small positive coefficient related to the hardness and the wear of the contact surface. (2.12), (2.13) represent the electric boundary conditions. Finally, in (2.14) $u_{0}$ is the given initial displacement, $v_{0}$ is the given initial velocity and $\theta_{0}$ is the initial temperature. 


\section{Variational formulation and preliminaries}

For a weak formulation of the problem, first we introduce some notation. The indices $i, j, k, l$ range from 1 to $d$ and summation over repeated indices is implied. An index that follows a comma represents the partial derivative with respect to the corresponding component of the spatial variable, e. g: $u_{i . j}=\frac{\partial u_{i}}{\partial x_{j}}$. We also use the following notations:

$$
\begin{aligned}
& H=\mathbb{L}^{2}(\Omega)^{d}=\left\{u=\left(u_{i}\right) / u_{i} \in \mathbb{L}^{2}(\Omega)\right\}, \mathcal{H}=\left\{\sigma=\left(\sigma_{i j}\right) / \sigma_{i j}=\sigma_{j i} \in \mathbb{L}^{2}(\Omega)\right\}, \\
& H_{1}=\left\{u=\left(u_{i}\right) / \varepsilon(u) \in \mathcal{H}\right\}, \mathcal{H}_{1}=\{\sigma \in \mathcal{H} / \text { Div } \sigma \in H\}
\end{aligned}
$$

The operators of deformation $\varepsilon$ and divergence Div are defined by

$$
\varepsilon(u)=\left(\varepsilon_{i j}(u)\right), \varepsilon_{i j}(u)=\frac{1}{2}\left(u_{i, j}+u_{j, i}\right), D i v \sigma=\left(\sigma_{i j, j}\right) .
$$

The spaces $H, \mathcal{H}, H_{1}$, and $\mathcal{H}_{1}$ are real Hilbert spaces endowed with the canonical inner products.

We denote by $|\cdot|_{H}$ (respectively; $|\cdot|_{\mathcal{H}},|\cdot|_{H_{1}}$, and $\left.|\cdot|_{\mathcal{H} 1}\right)$ the associated norm on the space $H$ ( respectively; $\mathcal{H}, H_{1}$, and $\left.\mathcal{H}_{1}\right)$.

We use standard notation for the $\mathbb{L}^{p}$ and the Sobolev spaces associated with $\Omega$ and $\Gamma$ and, for a function $\psi \in H^{1}(\Omega)$ we still write $\psi$ to denote it trace on $\Gamma$. We recall that the summation convention applies to a repeated index.

For the electric displacement field we use two Hilbert spaces:

$$
\mathcal{W}=\mathbb{L}^{2}(\Omega)^{d}, \quad \mathcal{W}_{1}=\left\{D \in \mathcal{W}, \operatorname{div} D \in \mathbb{L}^{2}(\Omega)\right\}
$$

endowed with the inner products:

$$
(D, E)_{\mathcal{W}}=\int_{\Omega} D_{i} E_{i} d x, \quad(D, E)_{\mathcal{W}_{1}}=(D, E)_{\mathcal{W}}+(\operatorname{div} D, \operatorname{div} E)_{\mathbb{L}^{2}(\Omega)} .
$$

And the associated norm $|\cdot|_{\mathcal{W}}\left(\right.$ respectively; $\left.|\cdot|_{\mathcal{W}_{1}}\right)$. The electric potential field is to be found in:

$$
W=\left\{\psi \in H^{1}(\Omega), \psi=0 \text { on } \Gamma_{a}\right\} .
$$

Since meas $\left(\Gamma_{a}\right)>0$, the following Friedrichs-Poincaré's inequality holds, thus:

$$
|\nabla \psi|_{\mathcal{W}} \geq c_{F}|\psi|_{H^{1}(\Omega)} \quad \forall \psi \in W
$$

where $c_{F}>0$ is a constant which depends only on $\Omega$ and $\Gamma_{a}$. On $W$, we use the inner product given by:

$$
(\varphi, \psi)_{W}=(\nabla \varphi, \nabla \psi)_{\mathcal{W}}
$$

and let $|\cdot|_{W}$ be the associated norm. It follows from $(3.1)$ that $|\cdot|_{H^{1}(\Omega)}$ and $|\cdot|_{W}$ are equivalent norms on $W$ and therefore $\left(W,|\cdot|_{W}\right)$ is a real Hilbert space.

Moreover, by the Sobolev trace Theorem, there exists a constant $\widetilde{c}_{0}$, depending only on $\Omega, \Gamma_{a}$ and $\Gamma_{3}$ such that:

$$
|\psi|_{L^{2}\left(\Gamma_{3}\right)} \leq \widetilde{c}_{0}|\psi|_{W} \quad \forall \psi \in W .
$$

We recall that when $D \in \mathcal{W}_{1}$ is a sufficiently regular function, the Green's type formula holds:

$$
(D, \nabla \psi)_{\mathcal{W}}+(\operatorname{div} D, \psi)_{\mathbb{L}^{2}(\Omega)}=\int_{\Gamma} D \nu \cdot \psi d a
$$


When $\sigma$ is a regular function, the following Green's type formula holds:

$$
(\sigma, \varepsilon(v))_{\mathcal{H}}+(D i v \sigma, v)_{H}=\int_{\Gamma} \sigma \nu . v d a \quad \forall v \in H_{1} .
$$

Next, we define the space:

$$
V=\left\{u \in H_{1} / u=0 \text { on } \Gamma_{1}\right\} .
$$

Since meas $\left(\Gamma_{1}\right)>0$, the following Korn's inequality holds:

$$
|\varepsilon(u)|_{\mathcal{H}} \geq c_{K}|v|_{H_{1}} \quad \forall v \in V
$$

where $c_{K}>0$ is a constant which depends only on $\Omega$ and $\Gamma_{1}$. On the space $V$ we use the inner product:

$$
(u, v)_{V}=(\varepsilon(u), \varepsilon(v))_{\mathcal{H}},
$$

let $|\cdot|_{V}$ be the associated norm. It follows by (3.5) that the norms $|\cdot|_{H_{1}}$ and $|\cdot|_{V}$ are equivalent norms on $V$ and therefore, $\left(V,|\cdot|_{V}\right)$ is a real Hilbert space. Moreover, by the Sobolev trace Theorem, there exists a constant $c_{0}$ depending only on the domain $\Omega, \Gamma_{1}$ and $\Gamma_{3}$ such that:

$$
|v|_{L^{2}\left(\Gamma_{3}\right)^{d}} \leq c_{0}|v|_{V} \quad \forall v \in V .
$$

In what follows, we assume the following assumptions on the problem $P$.

$$
\begin{aligned}
& \left\{\begin{array}{l}
(a): \mathcal{A}: \Omega \times \mathbb{S}^{d} \rightarrow \mathbb{S}^{d}, \\
(b): \exists M_{\mathcal{A}}>0 \text { such that }:\left|\mathcal{A}\left(x, \varepsilon_{1}\right)-\mathcal{A}\left(x, \varepsilon_{2}\right)\right| \leq M_{\mathcal{A}}\left|\varepsilon_{1}-\varepsilon_{2}\right| \\
\forall \varepsilon_{1}, \varepsilon_{2} \in \mathbb{S}^{d}, \text { a. } e . x \in \Omega, \\
(c): \exists m_{\mathcal{A}}>0 \text { such that }:\left|\mathcal{A}\left(x, \varepsilon_{1}\right)-\mathcal{A}\left(x, \varepsilon_{2}\right), \varepsilon_{1}-\varepsilon_{2}\right| \geq m_{\mathcal{A}}\left|\varepsilon_{1}-\varepsilon_{2}\right|^{2} \\
\forall \varepsilon_{1}, \varepsilon_{2} \in \mathbb{S}^{d}, \text { a. } e . x \in \Omega, \\
(d): \text { the mapping } x \rightarrow \mathcal{A}(x, \varepsilon) \text { is lebesgue measurable in } \Omega \text { for all } \varepsilon \in \mathbb{S}^{d}, \\
(e): \text { the mapping } x \rightarrow \mathcal{A}(x, 0) \in \mathcal{H},
\end{array}\right. \\
& \left\{\begin{aligned}
(a): \mathcal{G}: \Omega \times \mathbb{S}^{d} \rightarrow \mathbb{S}^{d}, \\
(b): \exists M_{\mathcal{G}}>0 \text { such that }:\left|\mathcal{G}\left(x, \xi_{1}\right)-\mathcal{G}\left(x, \xi_{2}\right)\right| \leq M_{\mathcal{G}}\left|\xi_{1}-\xi_{2}\right| \\
\forall \xi_{1}, \xi_{2} \in \mathbb{S}^{d}, \text { a. e. } x \in \Omega, \\
(d): \text { the mapping } x \rightarrow \mathcal{G}(x, \xi) \text { is lebesgue measurable in } \Omega \text { for all } \xi \in \mathbb{S}^{d}, \\
(e): \text { the mapping } x \rightarrow \mathcal{G}(x, 0) \in \mathcal{H},
\end{aligned}\right. \\
& \left\{\begin{array}{l}
(a): \xi=\left(e_{i j k}\right): \Omega \times \mathbb{S}^{d} \rightarrow \mathbb{R}^{d}, \\
(b): \xi(x, \tau)=\left(e_{i j k}(x) \tau_{j k}\right) \forall \tau=\left(\tau_{i j}\right) \in \mathbb{S}^{d}, \text { a. e. } x \in \Omega, \\
(c): e_{i j k}=e_{i k j} \in \mathbb{L}^{\infty}(\Omega),
\end{array}\right. \\
& \left\{\begin{array}{l}
(a): \beta=\left(\beta_{i j}\right): \Omega \times \mathbb{R}^{d} \rightarrow \mathbb{R}^{d}, \\
(b): \beta(x, E)=\left(b_{i j}(x) E_{j}\right) \forall E=\left(E_{i}\right) \in \mathbb{R}^{d}, \text { a.e.x } \in \Omega, \\
(c): b_{i j}=b_{j i} \in \mathbb{L}^{\infty}(\Omega), \\
(d): \exists m_{\beta}>0 \text { such that }: b_{i j}(x) E_{i} E_{j} \geq m_{\beta}|E|^{2} \\
\forall E=\left(E_{i}\right) \in \mathbb{R}^{d}, x \in \Omega .
\end{array}\right.
\end{aligned}
$$

From the assumptions (3.9) and (3.10),we deduce that the piezoelectric operator $\xi$ (respectively; the electric permittivity operator $\beta$ ) is linear, has measurable bounded 
component denoted $e_{i j k}$ ( respectively; $b_{i j}$ ) and moreover, $\beta$ is symmetric and positive definite.

Recall also that the transposed operator $\xi^{*}$ is given by $\xi^{*}=\left(e_{i j k}^{*}\right)$ where $e_{i j k}^{*}=e_{k i j}$ and the following equality holds:

$$
\xi \sigma . v=\sigma \cdot \xi^{*} v \quad \forall \sigma \in \mathbb{S}^{d}, \quad v \in \mathbb{R}^{d} .
$$

The friction function satisfies:

$$
\left\{\begin{array}{l}
p: \Gamma_{3} \times \mathbb{R} \rightarrow \mathbb{R}_{+} \text {verifies: } \\
(a): \exists M>0 \text { such that }:\left|p\left(x, r_{1}\right)-p\left(x, r_{2}\right)\right| \leq M\left|r_{1}-r_{2}\right| \\
\text { For every } r_{1}, r_{2} \in \mathbb{R}, a . e . x \in \Gamma_{3}, \\
(b): \text { the mapping }: x \rightarrow p(x, r) \text { is measurable on } \Gamma_{3} \text {, for every } r \in \mathbb{R}, \\
(c): p(x, 0)=0, \text { a. e. } x \in \Gamma_{3} .
\end{array}\right.
$$

We note that (3.11) is satisfied in the case in which $p$ given by (2.12).

We also assume that the body forces and surface tractions have the regularity:

$$
f_{0} \in \mathbb{L}^{2}(0 . T ; H), \quad h \in \mathbb{L}^{2}\left(0 . T ; \mathbb{L}^{2}\left(\Gamma_{2}\right)^{d}\right),
$$

The thermal tensors and the heat source density satisfy

$$
M=\left(m_{i j}\right), m_{i j}=m_{j i} \in L^{\infty}(\Omega), q_{e} \in L^{2}\left(0, T ; L^{2}(\Omega)\right),
$$

and for some $c_{k}>0$, for all $\left(\zeta_{i}\right) \in R_{d}$ :

$$
k=\left(k_{i, j}\right), k_{i j}=k_{j i} \in L^{\infty}(\Omega), k_{i j} \zeta_{i} \zeta_{j} \geq c_{k} \zeta_{i} \zeta_{j}
$$

as well as the densities of electric charges satisfy:

$$
q_{0} \in \mathbb{L}^{2}\left(0 . T ; \mathbb{L}^{2}(\Omega)\right), \quad q_{2} \in \mathbb{L}^{2}\left(0 . T ; \mathbb{L}^{2}\left(\Gamma_{b}\right)\right) .
$$

We define the function $f:[0 . T] \rightarrow V$ and $q:[0 . T] \rightarrow W$ by:

$$
\begin{aligned}
& (f(t), v)_{V}=\int_{\Omega} f_{0}(t) v d x+\int_{\Gamma_{2}} h(t) v d a \quad \forall v \in V, \quad t \in[0 . T], \\
& (q(t), \psi)_{W}=-\int_{\Omega} q_{0}(t) \psi d x+\int_{\Gamma_{b}} q_{2}(t) \psi d a \quad \forall \psi \in W, \quad t \in[0 . T] .
\end{aligned}
$$

for all $u, v \in V, \psi \in W$ and $t \in[0 . T]$, and note that conditions (3.14) and (3.15) imply that

$$
f \in \mathbb{L}^{2}\left(0 . T ; V^{\prime}\right), \quad q \in \mathbb{L}^{2}(0 . T ; W),
$$

while the friction coefficient $\mu$, the mass density $\rho$ satisfies

$$
\begin{aligned}
& \mu \in \mathbb{L}^{\infty}\left(\Gamma_{3}\right), \mu(x) \geq 0, a . e . \text { on } \Gamma_{3}, \\
& \rho \in \mathbb{L}^{\infty}(\Omega) \text { there exists } \rho^{*}>0 \text { such that } \rho(x) \geq \rho^{*}, \text { a.e. } x \in \Omega . \\
& u_{0} \in V, \quad v_{0} \in H, \theta_{0} \in E, \theta_{R} \in W^{1,2}\left(0, T ; L^{2}\left(\Gamma_{3}\right)\right), k_{e} \in L^{\infty}\left(\Omega, \mathbb{R}_{+}\right),
\end{aligned}
$$

The function $r: V \rightarrow L_{2}(\Omega)$ satisfies that there exists a constant $L_{r}>0$ such that

$$
\left|r\left(v_{1}\right)-r\left(v_{2}\right)\right|_{L 2(\Omega)} \leq L_{r}\left|v_{1}-v_{2}\right|_{V}, \forall v_{1}, v_{2} \in V
$$

We denote by the frictionfunctional $j: \mathcal{H} \times V \rightarrow \mathbb{R}$

$$
j(\sigma, v)=\int_{\Gamma_{3}} \mu p\left|R \sigma_{\nu}\right|\left|v_{\tau}\right| d a .
$$


We denote by $U$ the convex subset of admissible displacements fields given by

$$
U=\left\{v \in H_{1} / v=0 \text { on } \Gamma_{1}, v_{\nu} \leq 0 \text { on } \Gamma_{3}\right\},
$$

By a standard procedure based on Green's formula, we obtain the following formulation of the mechanical problem $(2.1)-(2.14)$.

Problem PV. Find a displacement field $u: \Omega \times[0 . T] \rightarrow \mathbb{R}^{d}$, an electric potentiel field $\varphi: \Omega \times[0 . T] \rightarrow \mathbb{R}$, an electric displacement field $D: \Omega \times[0 . T] \rightarrow \mathbb{R}^{d}$ such that and a temprature field $\theta: \Omega \times[0, T] \rightarrow \mathbb{R}_{+}$such that:

$$
\begin{gathered}
(\ddot{u}, w-\dot{u})_{V^{\prime} \times V}+(\sigma(t), \varepsilon(w-\dot{u}(t)))_{\mathcal{H}}+j(\sigma, w)-j(\sigma, \dot{u}(t)) \\
\geq(f(t), w-\dot{u}(t)) \forall u, w \in V \\
(D(t), \nabla \psi)_{\mathbb{L}^{2}(\Omega)^{d}}+(q(t), \psi)_{W}=0 \quad \forall \psi \in W \\
\left(\theta \dot{(t)}+K \theta(t)=R_{e} \dot{u}(t)+Q(t) \quad t \in(0, T), \quad \forall \psi \in W\right. \\
u(0)=u_{0}, \dot{u}(0)=v_{0} \text { and } \theta(0)=\theta_{0}
\end{gathered}
$$

where $Q:[0, T] \rightarrow E^{\prime}, K: E \rightarrow E^{\prime}, R: V \rightarrow E^{\prime}$ are given by

$$
\begin{aligned}
&(Q(t), \mu)_{W}=\int_{\Gamma_{3}} k_{e}\left(u_{\nu}\right) \theta_{R} \mu d a+\int_{\Omega} q \mu d x \\
&(K \tau, \mu)_{E^{\prime} \times E}=\sum_{i, j=1}^{d} \int_{\Omega} k_{i j} \frac{\partial \tau}{\partial x_{j}} \frac{\partial \mu}{\partial x_{i}} d x+\int_{\Gamma_{3}} k_{e} \tau \mu d a,\left(R_{e} \mu, v\right)_{E^{\prime} \times E} \\
&=-\int_{\Omega}(M \nabla v) d x
\end{aligned}
$$

for all $v \in V, \mu, \tau \in E$.

\section{Existence and uniqueness result}

Our main result which states the unique solvability of Problem are the following. Theorem 4.1. Let the assumptions (3.7)-(3.20) hold. Then, Problem PV has a unique solution $\{u, \varphi, D, \theta\}$ which satisfies

$$
\begin{aligned}
& u \in C^{1}(0 . T ; H) \cap W^{1.2}(0 . T ; V) \cap W^{2.2}\left(0 . T ; V^{\prime}\right) \\
& \varphi \in W^{1.2}(0 . T ; W) \\
& \sigma \in \mathbb{L}^{2}(0 . T ; \mathcal{H}), D i v \sigma \in \mathbb{L}^{2}\left(0 . T ; V^{\prime}\right) \\
& D \in W^{1.2}\left(0 . T ; \mathcal{W}_{1}\right) \\
& \theta \in W^{1,2}\left(0, T ; E^{\prime}\right) \cap L^{2}(0, T ; E) \cap C\left(0, T ; L^{2}(\Omega)\right)
\end{aligned}
$$

We conclude that under the assumptions (3.7) - (3.21), the mechanical problem (2.1) (2.14) has a unique weak solution with the regularity $(4.1)-(4.5)$. The proof of this theorem will be carried out in several steps. It is based on arguments of first order evolution nonlinear inequalities (see Refs. [5,7-9]), evolution equations (see Ref. [2]), and fixed point arguments. 
Let $G \in \mathbb{L}^{2}(0 . T ; \mathcal{H})$ and $\eta \in \mathbb{L}^{2}\left(0 . T ; V^{\prime}\right)$ are given, we deduce a variational formulation of Problem $P V$.

Problem $P V_{G \eta}$ : Find a displacement field $u_{G \eta}:[0 . T] \rightarrow V$ such that

$$
\begin{gathered}
\left\{\begin{array}{c}
u_{G \eta}(t) \in U \quad\left(\ddot{u}_{G \eta}, w-\dot{u}_{G \eta}\right)_{V^{\prime} \times V}+\left(\mathcal{A} \varepsilon\left(\dot{u}_{G \eta}(t)\right), \varepsilon\left(w-\dot{u}_{G \eta}(t)\right)_{\mathcal{H}}+\right. \\
\left(\eta, w-\dot{u}_{G \eta}(t)\right)_{V^{\prime} \times V}+j(G, w)-j\left(G, \dot{u}_{G \eta}(t)\right) \geq\left(f(t), w-\dot{u}_{G \eta}(t)\right) \\
\forall w \in V
\end{array}\right. \\
\dot{u}_{G \eta}(0)=v(0)=v_{0}
\end{gathered}
$$

We define $f_{\eta}(t) \in V$ for a.e.t $\in[0 . T]$ by

$$
\left(f_{\eta}(t), w\right)_{V^{\prime} \times V}=(f(t)-\eta(t), w)_{V^{\prime} \times V}, \forall w \in V .
$$

From (3.18), we deduce that:

$$
f_{\eta} \in \mathbb{L}^{2}\left(0 . T ; V^{\prime}\right)
$$

Let now $u_{G \eta}:[0 . T] \rightarrow V$ be the function defined by

$$
u_{G \eta}(t)=\int_{0}^{t} v_{G \eta}(s) d s+u_{0}, \quad \forall t \in[0 . T]
$$

We define the operator $A: V^{\prime} \rightarrow V$ by

$$
(A v, w)_{V^{\prime} \times V}=(\mathcal{A} \varepsilon(v), \varepsilon(w))_{\mathcal{H}}, \forall v, w \in V .
$$

Lemma 4.2. For all $G \in \mathbb{L}^{2}(0 . T ; \mathcal{H})$ and $\eta \in \mathbb{L}^{2}\left(0 . T ; V^{\prime}\right), P V_{G \eta}$ has a unique solution with the regularity:

$$
v_{G \eta} \in C(0 . T ; H) \cap \mathbb{L}^{2}(0 . T ; V) \text { and } \dot{v}_{G \eta} \in \mathbb{L}^{2}\left(0 . T ; V^{\prime}\right) .
$$

Proof. The proof from nonlinear first order evolution inequalities, given in Refs $([8])$.

In the second step, we use the displacement field $u_{G \eta}$ to consider the following variational problem.

Problem $P V 1_{G \eta}$ : Find an electric potential field $\varphi_{G \eta}: \Omega \times[0 . T] \rightarrow W$ such that:

$$
\begin{aligned}
\left(\beta \nabla \varphi_{G \eta}(t), \nabla \psi\right)_{\mathbb{L}^{2}(\Omega)^{d}}-\left(\xi \varepsilon\left(u_{G \eta}(t)\right), \nabla \psi\right)_{\mathbb{L}^{2}(\Omega)^{d}} & =(q(t), \psi)_{W} \\
\forall \psi & \in W, t \in[0 . T]
\end{aligned}
$$

We have the following result for $P V 1_{G \eta}$ :

Lemma 4.3. There exists a unique solution $\varphi_{G \eta} \in W^{1.2}(0 . T ; W)$ satisfies (4.13), moreover if $\varphi_{1}$ and $\varphi_{2}$ are two solutions to (4.13). Then, there exists a constants $c>0$ sach that:

$$
\left|\varphi_{1}(t)-\varphi_{2}(t)\right|_{W} \leq c\left|u_{1}(t)-u_{2}(t)\right|_{V} \forall t \in[0 . T] .
$$

Proof. See [16]. 
In the third step, we use the displacement field $u_{\eta}$ obtained in Lemma 4.2 to consider the following variational problem.

Problem $P V 1_{\theta \eta}$ : Find $\theta_{\eta}:[0, T] \rightarrow E^{\prime}$ satisfying a.e. $t \in(0, T)$

$$
\begin{aligned}
\theta_{\eta}(t)+K \theta_{\eta}(t) & =R_{e} \dot{u_{\eta}}(t)+Q(t) \quad t \in(0, T), \text { in } E^{\prime}, \\
\theta_{\eta}(0) & =\theta_{0} .
\end{aligned}
$$

Lemma 4.4. Problem $P V 1_{\theta \eta}$ has a unique solution, for all $\eta \in \mathcal{W}$,

$$
\theta_{\eta} \in W^{1,2}\left(0 ; T ; E^{\prime}\right) \cap L^{2}(0 ; T ; E) \cap C\left(0 ; T ; L^{2}(\Omega)\right), \quad C>0, \forall \eta \in L^{2}\left(\bar{I} ; V^{\prime}\right)
$$

satisfying

$$
\left|\theta_{\eta_{1}}-\theta_{\eta_{2}}\right|_{L^{2}(\Omega)}^{2} \leq C \int_{0}^{T}\left|v_{1}(s)-v_{2}(s)\right|_{V}^{2} d s \quad \forall t \in(0, T) .
$$

Proof. The existence and uniqueness result verifying (4.15) follows from classical result on first order evolution equation, applied to the Gelfand evolution triple

$$
E \subset F \equiv F^{\prime} \subset E^{\prime}
$$

We verify that the operator $K$ is linear, continuous, strongly monotone, and from the expression of the operator $R, v_{\eta} \in W^{1,2}(0, T ; V) \Rightarrow R v_{\eta} \in W^{1,2}(0, T ; F)$, as $Q \in W^{1,2}(0, T ; E)$ then $R v_{\eta}+Q \in W^{1,2}(0, T ; E)$. We deduce (4.17) see [1].

We consider the operator

$$
\begin{gathered}
\Lambda: \mathbb{L}^{2}\left(0 . T ; \mathcal{H} \times V^{\prime}\right) \rightarrow \mathbb{L}^{2}\left(0 . T ; \mathcal{H} \times V^{\prime}\right) \text { be defined as } \\
\Lambda(G, \eta)=\left(\Lambda_{1}(G), \Lambda_{2}(\eta)\right), \forall G \in \mathbb{L}^{2}(0 . T ; \mathcal{H}), \forall \eta \in \mathbb{L}^{2}\left(0 . T ; V^{\prime}\right), \\
\left|\Lambda\left(G_{2}, \eta_{2}\right)-\Lambda\left(G_{1}, \eta_{2}\right)\right|^{2}=\left|\left(\Lambda_{1}\left(G_{2}\right), \Lambda_{2}\left(\eta_{2}\right)\right)-\left(\Lambda_{1}\left(G_{1}\right), \Lambda_{2}\left(\eta_{1}\right)\right)\right|^{2} \\
\left|\Lambda_{1}\left(G_{2}\right)-\Lambda_{1}\left(G_{1}\right), \Lambda_{2}\left(\eta_{2}\right)-\Lambda_{2}\left(\eta_{1}\right)\right|^{2}=\left|\Lambda_{1}\left(G_{2}\right)-\Lambda_{1}\left(G_{1}\right)\right|^{2} \\
+\left|\Lambda_{2}\left(\eta_{2}\right)-\Lambda_{2}\left(\eta_{1}\right)\right|^{2} .
\end{gathered}
$$

We show that $\Lambda$ has a unique fixed point.

\section{Lemma 4.5.}

$$
\Lambda\left(G^{*}, \eta^{*}\right)=\left(G^{*}, \eta^{*}\right)
$$

Proof. Let $\left(G_{i}, \eta_{i}\right)$ are functions in $\mathbb{L}^{2}\left(0 . T ; \mathcal{H} \times V^{\prime}\right)$ and denote by $\left(u_{i}, \varphi_{i}, \theta_{i}\right)$ the functions obtained in Lemma 4.2, Lemma 4.3 and Lemma 4.4, for $(G, \eta)=\left(G_{i}, \eta_{i}\right) i=1.2$. Let $t \in[0 . T]$. From (2.1) it results

$$
\begin{aligned}
&\left|G_{2}-G_{1}\right|_{\mathcal{H}}^{2} \leq c\left(\left|v_{2}(t)-v_{1}(t)\right|_{V}^{2}+\left|\varphi_{2}(t)-\varphi_{1}(t)\right|_{W}^{2}\right. \\
&\left.+\left|u_{2}(t)-u_{1}(t)\right|_{V}^{2}+\left|\theta_{\eta_{1}}-\theta_{\eta_{2}}\right|_{L^{2}(\Omega)}^{2}\right)
\end{aligned}
$$

Therefore (4.14) and (4.17) yields

$$
\left|G_{2}-G_{1}\right|_{\mathcal{H}}^{2} \leq c\left(\left|v_{2}(t)-v_{1}(t)\right|_{V}^{2}+\left|u_{2}(t)-u_{1}(t)\right|_{V}^{2}+\int_{0}^{T}\left|v_{1}(s)-v_{2}(s)\right|_{V}^{2} d s\right) \text {. }
$$


Using (4.6),we find

$$
\begin{aligned}
& \left(\dot{v}_{2}(t)-\dot{v}_{1}(t), v_{2}(t)-v_{1}(t)\right)+\left(\mathcal{A} \varepsilon\left(v_{2}(t)\right)-\mathcal{A} \varepsilon\left(v_{1}(t)\right), v_{2}(t)-v_{1}(t)\right)+ \\
& \left(\eta_{2}(t)-\eta_{1}(t), v_{2}(t)-v_{1}(t)\right)+j\left(G_{2}, v_{2}(t)\right)-j\left(G_{2}, v_{1}(t)\right) \\
& -j\left(G_{1}, v_{2}(t)\right)+j\left(G_{1}, v_{1}(t)\right) \leq 0
\end{aligned}
$$

And, we have

$$
\begin{aligned}
j\left(G_{2}, v_{2}(t)\right)-j\left(G_{2}, v_{1}(t)\right)-j\left(G_{1}, v_{2}(t)\right)+j\left(G_{1}, v_{1}(t)\right) \\
\leq \int_{\Gamma_{3}} \mu p\left|R G_{2 \nu}\right|\left|v_{2 \tau}\right| d a-\int_{\Gamma_{3}} \mu p\left|R G_{2 \nu}\right|\left|v_{1 \tau}\right| d a \\
\quad-\int_{\Gamma_{3}} \mu p\left|R G_{1 \nu}\right|\left|v_{2 \tau}\right| d a+\int_{\Gamma_{3}} \mu p\left|R G_{1 \nu}\right|\left|v_{1 \tau}\right| d a .
\end{aligned}
$$

Moreover, from (3.11), (3.19) and using the properties of $R$, we find

$$
j\left(G_{2}, v_{2}(t)\right)-j\left(G_{2}, v_{1}(t)\right)-j\left(G_{1}, v_{2}(t)\right)+j\left(G_{1}, v_{1}(t)\right) \leq c\left|G_{2}-G_{1}\right|_{\mathcal{H}}\left|v_{2}-v_{1}\right|_{V}
$$

So, (4.22) will be

$$
\begin{aligned}
& \left(\dot{v}_{2}(t)-\dot{v}_{1}(t), v_{2}(t)-v_{1}(t)\right)_{V^{\prime} \times V}+\left(\mathcal{A} \varepsilon\left(v_{2}(t)\right)-\mathcal{A} \varepsilon\left(v_{1}(t)\right), v_{2}(t)-v_{1}(t)\right) \\
& +\left(\eta_{2}(t)-\eta_{1}(t), v_{2}(t)-v_{1}(t)\right) \leq c\left|G_{2}-G_{1}\right|_{\mathcal{H}}\left|v_{2}-v_{1}\right|_{V}
\end{aligned}
$$

We integrate this equality with respect to time.

We use the initial conditions $v_{1}(0)=v_{2}(0)=v_{0}$, the relation (3.7) and CauchySchwarz's inequality. for all $t \in[0, T]$. Then, using the inequality

$$
2 a b \leq \frac{a^{2}}{m_{\mathcal{A}}}+m_{\mathcal{A}} b^{2},
$$

we obtain

$$
\begin{aligned}
& \frac{1}{2}\left|v_{2}(t)-v_{1}(t)\right|_{V}^{2}+\frac{m_{\mathcal{A}}}{2} \int_{0}^{t}\left|v_{2}(s)-v_{1}(s)\right|_{V}^{2} d s \\
& \leq \frac{1}{2 m_{\mathcal{A}}} \int_{0}^{t}\left|\eta_{2}(s)-\eta_{1}(s)\right|_{V^{\prime}}^{2}+\frac{m_{\mathcal{A}}}{2} \int_{0}^{t}\left|v_{2}(s)-v_{1}(s)\right|_{V}^{2} d s \\
& +c\left(\int_{0}^{t}\left|G_{2}(s)-G_{1}(s)\right|_{\mathcal{H}}^{2}+\int_{0}^{t}\left|v_{2}(s)-v_{1}(s)\right|_{V}^{2} d s .\right)
\end{aligned}
$$

We apply Gronwall's inequality to obtain

$$
\left|v_{2}(t)-v_{1}(t)\right|_{V}^{2} \leq c\left(\int_{0}^{t}\left|G_{2}(s)-G_{1}(s)\right|_{\mathcal{H}}^{2} d s+\int_{0}^{t}\left|\eta_{2}(s)-\eta_{1}(s)\right|_{V^{\prime}}^{2}\right) .
$$

In other hand

$$
\left|\eta_{2}(t)-\eta_{1}(t)\right|_{V^{\prime}}^{2} \leq c\left(\left|\varphi_{2}(t)-\varphi_{1}(t)\right|_{W}^{2}+\left|u_{2}(t)-u_{1}(t)\right|_{V}^{2}+\left|\theta_{\eta_{1}}-\theta_{\eta_{2}}\right|_{L^{2}(\Omega)}^{2}\right)
$$

Therefore (4.14) and (4.17) yields

$$
\left|\eta_{2}(t)-\eta_{1}(t)\right|_{V^{\prime}}^{2} \leq c\left(\int_{0}^{T}\left|v_{1}(s)-v_{2}(s)\right|_{V^{2}}^{2} d s\right)
$$


Using (4.6), we find

$$
\begin{aligned}
& \left(\dot{v}_{2}(t)-\dot{v}_{1}(t), v_{2}(t)-v_{1}(t)\right)+\left(\mathcal{A} \varepsilon\left(v_{2}(t)\right)-\mathcal{A} \varepsilon\left(v_{1}(t)\right), v_{2}(t)-v_{1}(t)\right) \\
& +\left(\eta_{2}(t)-\eta_{1}(t), v_{2}(t)-v_{1}(t)\right)+j\left(G_{2}, v_{2}(t)\right)-j\left(G_{2}, v_{1}(t)\right) \\
& -j\left(G_{1}, v_{2}(t)\right)+j\left(G_{1}, v_{1}(t)\right) \leq 0
\end{aligned}
$$

We integrate this equality with respect to time.

We use the initial conditions $v_{1}(0)=v_{2}(0)=v_{0}$, the relations $(3.7),(4.24)$ and Cauchy-Schwarz's inequality, for all $t \in[0, T]$. Then, using the inequality

$$
a b \leq c\left(a^{2}+b^{2}\right)
$$

we obtain

$$
\int_{0}^{t}\left|v_{2}(s)-v_{1}(s)\right|_{V}^{2} d s \leq c\left(\int_{0}^{t}\left|\eta_{2}-\eta_{1}\right|_{V^{\prime}}^{2} d s+\int_{0}^{t}\left|G_{2}-G_{1}\right|_{\mathcal{H}}^{2} d s\right)
$$

Applying the inequality (4.10) in (4.31). So (4.21) will be

$$
\left|G_{2}(t)-G_{1}(t)\right|_{\mathcal{H}}^{2} \leq c\left(\int_{0}^{t}\left|\eta_{2}(s)-\eta_{1}(s)\right|_{V^{\prime}}^{2} d s+\int_{0}^{t}\left|G_{2}(s)-G_{1}(s)\right|_{\mathcal{H}}^{2} d s\right) .
$$

From (4.10), (4.29) and (4.31) we find

$$
\left|\eta_{2}-\eta_{1}\right|_{V^{\prime}}^{2} \leq c\left(\int_{0}^{t}\left|\eta_{2}-\eta_{1}\right|_{V^{\prime}}^{2} d s+\int_{0}^{t}\left|G_{2}-G_{1}\right|_{\mathcal{H}}^{2} d s\right)
$$

Using (4.16), to see that

$$
\left|\Lambda\left(G_{2}, \eta_{2}\right)-\Lambda\left(G_{1}, \eta_{1}\right)\right|^{2} \leq c \int_{0}^{t}\left|\left(G_{2}, \eta_{2}\right)-\left(G_{1}, \eta_{1}\right)\right|_{\mathcal{H} \times V^{\prime}}^{2} d s .
$$

And denoting by $p$ the powers of operator $\Lambda,(4.32)$ imply by recurrence that

$$
\begin{aligned}
\mid \Lambda^{p}\left(G_{2},\right. & \left.\eta_{2}\right)-\left.\Lambda^{p}\left(G_{1}, \eta_{1}\right)\right|_{L^{2}\left(0 . T ; \mathcal{H} \times V^{\prime}\right)} ^{2} \\
& \leq \frac{(c t)^{p}}{p !}\left|\left(G_{2}, \eta_{2}\right)-\left(G_{1}, \eta_{1}\right)\right|_{L^{2}\left(0 . T ; \mathcal{H} \times V^{\prime}\right)}^{2} .
\end{aligned}
$$

This inequality shows that for a sufficiently large $p$ the operator $\Lambda^{p}$ is a contraction on the Banach space $\mathbb{L}^{2}\left(0 . T ; \mathcal{H} \times V^{\prime}\right)$ and therefor, there exists a unique element: $\left(G^{*}, \eta^{*}\right) \in \mathbb{L}^{2}\left(0 . T ; \mathcal{H} \times V^{\prime}\right)$ such that

$$
\Lambda\left(G^{*}, \eta^{*}\right)=\left(G^{*}, \eta^{*}\right) .
$$

From (4.18), we find

$$
\left(G^{*}, \eta^{*}\right)=\left(\sigma_{G^{*}} \eta^{*}, \xi^{*} \nabla \varphi_{G^{*} \eta^{*}}+\mathcal{G} \varepsilon\left(u_{G^{*} \eta^{*}}\right)-\theta_{G^{*} \eta^{*}} M_{e}\right) .
$$

Now, we have all the ingredients to provide the proof of Theorem 4.1. 
Proof of Theorem 4.1. Existence. Let $\left(G^{*}, \eta^{*}\right) \in \mathbb{L}^{2}\left(0 . T ; \mathcal{H} \times V^{\prime}\right)$ be the fixed point of $P V_{G \eta}$ and let $\left(u^{*}, \varphi^{*}, \theta^{*}\right)$ be the solution to Problems $P V_{G \eta}, P V 1_{G \eta}$ and $P V 1_{\theta \eta}$ for $(G, \eta)=\left(G^{*}, \eta^{*}\right)$, that is, $u^{*}=u_{G^{*} \eta^{*}}, \varphi^{*}=\varphi_{G^{*}} \eta^{*}$ and $\theta^{*}=\theta_{G^{*} \eta^{*}}$. It results from $(3.24),(3.25)$ and $(3.26)$ that $\left(u^{*}, \varphi^{*}, \theta^{*}\right)$ is a solution of Problem $P V$. Property (4.1) (4.2) and (4.5) follows from Lemmas 4.2, 4.3 and 4.4.

Uniqueness. The uniqueness of the solution is a consequence of the uniqueness of the fixed point of operator defined by (4.18).

Acknowledgment. This work has been realized thanks to the: Direction générale de la Recherche Scientifique et du Développement Technologique "DGRSDT". MESRS Algeria. And Research Project under code: PRFUCOOL03UN1901200180004.

\section{References}

[1] Adly, S., Chau, O., On some dynamic thermal non clamped contact problems, Math. Programm., Ser. B, (2013), no. 139, 5-26.

[2] Barboteu, M., Fernández, J.R., Ouafik, Y., Numerical analysis of a frictionless viscoelastic piezoelectric contact problem, M2AN Math. Model. Numer. Anal., (in press).

[3] Barbu, V., Optimal Control of Variational Inequalities, Pitman, Boston, 1984.

[4] Batra, R.C., Yang,J.S., Saint Venant's principle in linear piezoelectricity, Journal of Elasticity, 38(1995), 209-218.

[5] Brézis, H., Analyse Fonctionnelle, Théorie et Application, Masson, Paris, 1987.

[6] Chau, O., On a class of second order evolution inéquality and application, Int. J. of Appl. Math. and Mech., 4(2008), no. 1, 24-48.

[7] Drabla, S., Zellagui, Z., Analysis of a electro-elastic contact problem with friction and adhesion, Stud. Univ. Babeş-Bolyai Math., 54(2009), 75-99.

[8] Duvaut G., Lions, J.L., Inequalities in Mechanics and Physics, Springer-Verlag, Berlin, 1988.

[9] Han, W., Sofonea, M., Kazmi, K., Analysis and numerical solution of a frictionless contact problem for electro-elastic-visco-plastic materials, Comput. Methods Appl. Mech. Engrg., 196(2007), 3915-3926.

[10] Jarusek, J., Sofonea, M. On the solvability of dynamic elastic-visco-plastic contact problems, Z. Angew. Math. Mech., 88(2008), 3-22.

[11] Mindlin, R.D., Polarisation gradient in elastic dielectrics, Int. J. Solids Structures, 4(1968), 637-663.

[12] Mindlin, R.D., Continuum and lattice theories of influence of electromechanical coupling on capaci- tance of thin dielectric films, Int. J. Solids, 4(1969), 1197-1213.

[13] Mindlin, R.D., Elasticity, Piezoelectricity and Cristal lattice dynamics, J. of Elasticity, 4 (1972), 217-280.

[14] Rochdi, M., Shillor, M., Sofonea, M., A quasistatic contact problem with directional friction and damped response, Appl. Anal., 68(1998), 409-422.

[15] Selmani, L., Bensebaa, N., An electro-viscoelastic contact problem with adhesion and damage, Rend. Semin. Mat. Univ. Politec. Torino, 66(2008), no. 2.

[16] Selmani, M., Selmani, L., Frictional contact problem for elastic-viscoplastic materials with thermal effect, Appl. Math. Mech. (English Ed.), 34(2013), no. 6, 761-776. 
[17] Sofonea, M., Essoufi, E.-H., Quasistatic frictional contact of a viscoelastic piezoelectric body, Adv. Math. Sci. Appl., 14(2004), no. 1, 25-40.

[18] Touzaline, A., A viscoelastic frictionless contact problem with adhesion, Boll. Pol. Acad. Sci. Math., 63(2015), 53-66.

\section{Sihem Smata}

Applied Mathematics Laboratory,

Department of Mathematics,

Faculty of Sciences, University of Setif 1,

19000, Algeria

e-mail: ssmata@yahoo.fr

Nemira Lebri

Applied Mathematics Laboratory,

Department of Mathematics, Faculty of Sciences,

University of Setif 1, 19000, Algeria

e-mail: nem_mat2000@yahoo.fr 